\title{
Categorización de la atención fonoaudiológica: una propuesta para adultos en contexto hospitalario
}

\author{
Axel Pavez ${ }^{\mathbf{a}, *}$, Bernardita Letelier-Vera ${ }^{\mathbf{a}}$, Carolina Rivera-Rivera ${ }^{\mathbf{a}, \mathbf{b}}$ \\ a Servicio de Medicina Física y Rehabilitación, Hospital de Urgencia Asistencia Pública, Chile \\ bervicio de Medicina Física y Rehabilitación, Clínica Alemana, Chile
}

\section{RESUMEN}

Existe la necesidad de desarrollar herramientas que demuestren el impacto de la intervención fonoaudiológica en el ámbito hospitalario, lo que exige implementar modelos asistenciales y de gestión, que favorezcan la demanda clínica y la eficiencia de los procesos y de los equipos. Diversas profesiones del área sanitaria han empleado modelos de categorización de la atención que, junto con satisfacer las demandas de los pacientes, traducen las actividades de su disciplina en términos de gestión. Actualmente, en el ámbito fonoaudiológico no se cuenta con herramientas de este tipo que permitan determinar la cantidad de atenciones necesarias para una persona en contexto hospitalario. Debido a lo anterior, en el artículo se presenta la creación, desarrollo y aplicación de una matriz cuali-cuantitativa. El objetivo de la matriz es valorar y estandarizar la carga de trabajo fonoaudiológico requerido para cada paciente adulto hospitalizado, considerando su contexto clínico global. Esta matriz de categorización considera cinco variables clínicas evaluadas durante la rehabilitación del paciente y cuatro calificadores en relación con el orden de severidad. Se establecen y proponen diferentes categorías de atención que entregan una carga terapéutica recomendada (desde 1 a 2 atenciones semanales en casos leves hasta 2 atenciones diarias en casos complejos). Finalmente, se discute su uso y los beneficios de su implementación como una herramienta que permite optimizar procesos de gestión, determinar brechas y justificar la inclusión de nuevos fonoaudiólogos en el contexto hospitalario.

\section{Categorizing speech-language pathology attention: a proposal for adults inpatient}

\section{ABSTRACT}

There is a need to develop tools that demonstrate the impact of speech therapy intervention in the hospital setting, which requires the implementation of care and management models that favor clinical demand and efficiency of processes and equipment. Various professions in the health area have used categorization models that, along with satisfying the demands of patients, translate the activities of their discipline into management terms. Currently, in the speech therapy field, there are no tools of this kind available that allow determining the amount of care necessary for a person in a hospital context. Due to the above circumstances, this article presents the creation, development and application of a qualitative-quantitative matrix. The objective of the matrix is to assess and standardize the speech therapy workload required for each hospitalized adult patient, considering their global clinical context. This categorization matrix considers five clinical variables evaluated during the patient's rehabilitation and four qualifiers in relation to the order of severity. Different categories of care that deliver a recommended therapeutic load (from 1 to 2 weekly visits in mild cases to 2 daily visits in complex cases) are established and proposed. Finally, their use and the benefits of their implementation are discussed as a tool that allows optimizing management processes, determining gaps and justifying the inclusion of new speech therapists in the hospital context.

\section{Palabras clave: \\ Administración hospitalaria; \\ Planificación sanitaria; \\ Calidad de la atención; \\ Fonoaudiología/organización \\ y administración}

*Autor/a correspondiente: Axel Pavez 


\section{INTRODUCCIÓN}

El modelo de atención multidisciplinario ha demostrado beneficios sobre la rehabilitación y los costos asociados a la atención en salud, tanto en Chile como en el mundo (HowardWilsher et al., 2016). Pese a ello, cada día se vuelve necesario para los profesionales demostrar el impacto de su intervención en las prácticas clínicas. Una de las formas de hacerlo es por medio del cálculo de indicadores, que objetiven los resultados y cambios que experimentan los pacientes durante su estadía hospitalaria (Duncan \& Murray, 2012).

En este sentido, el uso de herramientas de evaluación en la práctica clínica es relevante tanto para los profesionales de rehabilitación como para los pacientes, permitiendo así objetivar avances y resultados. El uso de escalas contribuye a mejorar el razonamiento, objetivar y cuantificar las intervenciones; lo cual es fundamental para mejorar los estándares de calidad de la atención (Gutiérrez-Panchana \& Hidalgo-Cabalín, 2018).

Los procesos de cambios en las demandas del sistema sanitario, las necesidades de los pacientes, el modelo de gestión y la atención en salud; exige que las instituciones y los equipos de salud adopten modelos asistenciales y de gestión que permitan favorecer las demandas clínicas y mejorar la eficiencia de los procesos y de los equipos de salud. Así es como diversas disciplinas (por ejemplo, Enfermería y Kinesiología) han empleado modelos de categorización que junto con satisfacer las demandas de los pacientes traducen las actividades de su disciplina en términos de gestión, identificando las necesidades de atención del usuario y definiendo niveles para ello (García \& Castillo, 2000; Landeros et al., 2014).

Uno de los principales problemas a los cuales se enfrentan los fonoaudiólogos en la atención cerrada (atención de hospitalizados) es la falta de herramientas clínicas que permitan objetivar el número de atenciones fonoaudiológicas que requiere cada paciente. Muchas veces es el médico tratante o interconsultor (Neurólogo, Otorrinolaringólogo, Fisiatra, Internista, Intensivista $\mathrm{u}$ otro) quien determina $\mathrm{y}$ asigna subjetivamente la carga de trabajo que requiere el paciente, sin considerar las diversas alteraciones fonoaudiológicas o la magnitud de las mismas. A ello se suma un déficit importante de recursos humanos fonoaudiológicos.

Actualmente en Chile, no existe un consenso para establecer la frecuencia de las atenciones fonoaudiológicas. Sin embargo, el documento "Modelo de Gestión Red de Rehabilitación" elaborado por el Departamento de Rehabilitación de la División de Gestión de Redes Asistenciales (DIGERA) del MINSAL (Ministerio de
Salud [MINSAL], 2018), propone entre otras cosas una fórmula para el cálculo de profesionales según número de camas hospitalarias y complejidad de las mismas. Plantea que, dependiendo del nivel de complejidad de la institución, se espera que sobre un $70 \%$ de las atenciones correspondan a atención cerrada con una frecuencia de 1 a 2 atenciones al día (MINSAL, 2018). Pese a ello, este valor es arbitrario y subjetivo, ya que carece de un método que permita objetivar la carga de atención.

Según nuestro conocimiento, no se cuenta con publicaciones a nivel nacional o internacional que propongan herramientas para determinar la carga terapéutica y la frecuencia de las atenciones. Por lo tanto, la implementación de un modelo de categorización de la atención, desde la Fonoaudiología, permitirá ordenar y jerarquizar las atenciones fonoaudiológicas basándose en las necesidades propias de cada paciente.

Todo lo previamente expuesto demuestra que se requiere contar con un proceso de gestión de la atención fonoaudiológica. Por ello, la Unidad de Fonoaudiología del Servicio de Medicina Física y Rehabilitación del Hospital de Urgencia Asistencia Pública (HUAP), ha elaborado e implementado una propuesta de categorización de la demanda fonoaudiológica.

El presente trabajo busca compartir el diseño y la aplicación de una herramienta de categorización, con el objetivo de valorar y estandarizar la carga de trabajo fonoaudiológico que requiere cada persona adulta hospitalizada, considerando su contexto clínico global.

\section{DESARROLLO}

\section{Contexto clínico e institucional}

El Hospital de Urgencia Asistencia Pública corresponde a un hospital público de alta complejidad, de atención terciaria, ubicado en la comuna de Santiago, Región Metropolitana, Chile. Es una de las principales instituciones públicas de salud y es líder en atención de pacientes críticos del país, siendo centro de referencia nacional de grandes quemados y centro de referencia regional para pacientes politraumatizados.

De acuerdo a datos no publicados del sistema institucional, la población del HUAP corresponde principalmente a personas adultas y adultos mayores, con una media de 54.8 años de edad y un rango etario que fluctúa desde los 17 años hasta los 103 años aproximadamente. Los diagnósticos más frecuentes corresponden a Politraumatismo, Traumatismo Encéfalo Craneano (TEC), 
Ataque Cerebro Vascular (ACV), Enfermedades Respiratorias, Quemaduras, Sepsis, entre otros.

Debido a la complejidad de la institución y su alta capacidad resolutiva en relación al manejo de patologías complejas, las atenciones de urgencia y de cuidados críticos son el fuerte de la institución, evidenciándose según datos del sistema de grupos relacionados por diagnóstico (GRD) una severidad por patología del doble del nivel nacional. Del mismo modo, la presión hacia el sistema público de salud por mantener un balance positivo en la ocupación de camas, fomenta la toma de decisiones rápidas en el actuar clínico fonoaudiológico; enfocándose en contribuir activamente en la disminución de días camas por complicaciones asociadas a la atención en salud y maximizar la eficiencia del trabajo fonoaudiológico.

Otro punto destacable es que en el HUAP la atención fonoaudiológica es netamente clínica, careciendo de evaluaciones objetivas como son la videofluoroscopía (VFC), video-deglución (FEES en inglés), nasofibroscopía (NFC), entre otras que contribuyen en el proceso de evaluación y terapia.

Es en este contexto que, en el año 2018, se crea la propuesta de categorización de la demanda fonoaudiológica. Es importante destacar que su elaboración implicó considerar las características de la institución y de la población que es atendida en ella. Por lo tanto, en caso de replicarla, se sugiere considerar estos aspectos.

\section{Matriz de categorización}

Para el diseño y construcción del modelo de categorización se utilizó una matriz de variables clínicas y su severidad, tomando como base lo propuesto por Gutiérrez-Panchana \& HidalgoCabalín (2018). El resultado final de la matriz de categorización puede ser revisado en el apartado Anexos, donde se encontrarán las variables y el puntaje asignado por severidad, así como ejemplos de uso.

En la Tabla 1 se presenta la matriz cuali-cuantitativa, que se compone por dos ejes. El eje horizontal considera 5 variables clínicas evaluadas durante la rehabilitación del paciente. Ellas fueron seleccionadas de acuerdo al perfil de los pacientes, los aspectos más relevantes son: la atención hospitalaria, la experiencia clínica del equipo y la evidencia disponible en relación a escalas de evaluación y severidad para pacientes hospitalizados.

Tabla 1. Diseño de la matriz de categorización.

\begin{tabular}{|c|c|c|c|c|c|}
\hline \multirow[t]{2}{*}{ Variables } & \multicolumn{4}{|c|}{ Calificadores } & \multirow[t]{2}{*}{ Puntaje } \\
\hline & $\begin{array}{c}\text { Calificador } \mathrm{W} \\
(0 \text { puntos })\end{array}$ & $\begin{array}{c}\text { Calificador } \mathrm{X} \\
\quad(1 \text { punto })\end{array}$ & $\begin{array}{c}\text { Calificador } Y \\
\text { (2 puntos) }\end{array}$ & $\begin{array}{c}\text { Calificador } \mathrm{Z} \\
\text { (3 puntos) }\end{array}$ & \\
\hline Variable Clínica A & & & & & $\mathrm{Pa}$ \\
\hline Variable Clínica B & & & & & $\mathrm{Pb}$ \\
\hline Variable Clínica C & & & & & $\mathrm{Pc}$ \\
\hline Variable Clínica D & & & & & $\mathrm{Pd}$ \\
\hline \multirow[t]{3}{*}{ Variable Clínica E } & & & & & $\mathrm{Pe}$ \\
\hline & \multirow{2}{*}{$\Sigma \mathrm{W}=0$} & $\operatorname{LiX} \leq \Sigma X \leq \operatorname{Ls} X$ & $\mathrm{LiY} \leq \Sigma \mathrm{Y} \leq \mathrm{LsY}$ & $\mathrm{LiZ} \leq \Sigma \mathrm{Z} \leq \mathrm{LsZ}$ & \multirow{2}{*}{$\mathrm{PT}^{(*)}$} \\
\hline & & $1 \leq \Sigma \mathrm{X} \leq 5$ & $2 \leq \Sigma Y \leq 10$ & $3 \leq \Sigma Z \leq 15$ & \\
\hline
\end{tabular}

$\Sigma \mathrm{W}$ : sumatoria de columna W; $\Sigma \mathrm{X}$ : sumatoria de columna X; $\Sigma \mathrm{Y}$ : sumatoria de columna Y; $\Sigma Z$ : sumatoria de columna Z; LiX: límite inferior X; LsX: límite superior X; LiY: límite inferior Y; LsY: límite superior Y; LiZ: límite inferior Z; LsZ: límite superior Z. ${ }^{(*)} \mathrm{PT}=\mathrm{Pa}+\mathrm{Pb}+\mathrm{Pc}+\mathrm{Pd}+\mathrm{Pe}$

Por su parte el eje vertical muestra 4 calificadores en orden de severidad; otorgando desde 0 puntos hasta 3 puntos en aquellos casos más severos. El menor calificador es la columna W, donde cada celda tiene un puntaje de 0 . El siguiente calificador es de la columna X, donde cada celda tiene un puntaje de 1. El calificador de la columna Y entrega 2 puntos en cada celda. Finalmente, el calificador de la columna $\mathrm{Z}$ es el de mayor severidad y entrega 3 puntos por cada celda.

El puntaje total observado es obtenido de la sumatoria $(\Sigma)$ de los puntajes obtenidos para cada una de las variables clínicas de 
acuerdo al calificador correspondiente; es decir, el PT es la suma de $\mathrm{Pa}+\mathrm{Pb}+\mathrm{Pc}+\mathrm{Pd}+\mathrm{Pe}$.

\section{Selección de las variables clínicas}

Las 5 variables clínicas seleccionadas para el sistema de categorización fueron:

1. Nivel de colaboración/respuesta: es la base de toda intervención activa, fundamental para las acciones terapéuticas, pues gran parte de los resultados dependen de ella. Para evaluar esta variable se consideró el Standardized Five Questions Scale (S5Q) (De Jonghe et al., 2002) que es una herramienta de cribado ampliamente utilizada en el contexto clínico, sobre todo en Unidades de Paciente Crítico (UPC). Consiste en 5 órdenes sencillas y estandarizadas, de menor a mayor nivel de complejidad, que permiten monitorizar rápidamente el nivel de colaboración y respuesta del paciente. Se considera que pacientes con un puntaje $\geq 3$ puntos presentan un nivel moderado de colaboración González-Seguel et al. (2017), que les permite continuar con el proceso de rehabilitación y aprendizaje.

2. Funcionamiento cognitivo: en aquellos sujetos que han sufrido una lesión cerebral es fundamental contar con criterios que permitan clasificar su nivel de funcionamiento cognitivo, pues las deficiencias cognitivas pueden interferir en la rehabilitación. Considerando el perfil de la población atendida en HUAP, la alta incidencia de TEC y ACV justifica la valoración del funcionamiento cognitivo. Por ello se consideró medir esta variable en aquellos pacientes que presenten alguna limitación en su funcionalidad cognitiva. Se incluyen en este grupo a pacientes trastornos cognitivos secundarios a TEC o ACV, pacientes con Trastornos Cognitivos-Comunicativos secundarios a diversas etiologías, entre otros. Se escogió la Escala de Rendimiento Cognitivo Rancho Los Amigos (ERLA), debido a que califica el nivel general de conciencia $\mathrm{y}$ funcionamiento cognitivo-conductual de un paciente con lesión cerebral. Si bien se ha utilizado mayoritariamente en pacientes con TEC, se usa comúnmente para clasificar a los pacientes con lesiones cerebrales en entornos de rehabilitación aguda y post-aguda, independiente de la etiología (Gouvier et al., 1987; Whyte \& Kunz, 2018).

3. Trastorno de deglución: la disfagia o trastorno de deglución es una condición frecuente en el contexto de pacientes hospitalizados. La alta morbi-mortalidad y los costos asociados a la atención en salud la posicionan como una variable de interés. Existen diversas escalas para clasificar y valorar la disfagia. De acuerdo a nuestra experiencia y al contexto clínico la Dysphagia Outcome and Severity Scale
(DOSS) (O’Neil et al., 1999) es la más apropiada, porque combina parámetros funcionales y estructurales. Además, se basa en los resultados de una valoración clínica; entregando recomendaciones para el tipo de alimentación según las características de cada caso.

4. Riesgo aspirativo: la penetración/aspiración laríngea son unos de los componentes más complejos de la disfagia orofaríngea, por lo que se consideran variables de interés. Los fonoaudiólogos clínicos miden regularmente la fisiopatología de la deglución y los mecanismos que subyacen a la penetración/aspiración. Tal como lo plantean Steele \& Cichero (2014) un énfasis importante de la evaluación de la deglución es identificar anormalidades en la fisiología deglutoria que contribuyan o expliquen el riesgo aspirativo de un paciente. En la matriz de categorización, se realiza una propuesta para valorar el riesgo aspirativo según los hallazgos fisiopatológicos y el criterio clínico. La propuesta se basó en la literatura (Daniels et al., 2000; O’Neil et al., 1999; Steele \& Cichero, 2014) y en la experiencia clínica, considerando los factores que interfieren de manera frecuente. Así, se establecen 3 niveles de riesgo aspirativo (alto, medio o bajo). Para mayor detalle, se sugiere revisar este apartado en el Anexo.

5. Comunicación: otro aspecto relevante desde el punto de vista fonoaudiológico es la comunicación. La presión hacia el sistema de atención cerrada de pacientes adultos genera la necesidad de priorizar a pacientes con disfagia por sobre aquellos con dificultades comunicativas. Esto ha significado que los fonoaudiólogos que se desempeñan en el contexto hospitalario presenten menores oportunidades para satisfacer las necesidades de aquellos pacientes con déficits comunicativos (Armstrong, 2003). Pese a este hecho, es fundamental detectar estas deficiencias. Así, en el contexto hospitalario, es posible pesquisar alteraciones comunicativas en periodo agudo o subagudo, que podrían involucrar dificultades de voz, habla, lenguaje y/o cognitivascomunicativas. Además del impacto en la funcionalidad comunicativa per se, estas alteraciones pueden repercutir a nivel psico-emocional y afectar la calidad de vida del individuo (O'Halloran et al., 2009; Radtke et al., 2011). Por tales motivos, en la propuesta de categorización, se incluyen como variables clínicas a calificar todos aquellos diagnósticos vinculados con alteraciones comunicativas.

Cabe destacar que, de las variables clínicas consideradas, sólo el S5Q ha sido utilizado en categorizaciones de otra disciplina (Gutiérrez-Panchana \& Hidalgo-Cabalín, 2018). Al no encontrarse información en la literatura sobre modelos de 
categorización fonoaudiológica, el resto de las variables seleccionadas se justifica según lo detallado en los párrafos previos.

\section{Categorías de atención}

Las categorías de atención determinan el nivel de demanda fonoaudiológica del paciente. Para la elaboración de las categorías de atención se consideró la información de la matriz expuesta en la Tabla 1, en donde por cada calificador (columna W, X, Y y Z) se realizó la sumatoria $(\Sigma)$ con el valor mínimo y máximo a obtener por cada categoría. Lo anterior si se considera al menos una celda con ese calificador para el valor mínimo y máximo las cinco celdas para el valor máximo. Esto implica el rango de puntaje, que fue calculado tomando como base el límite inferior y el límite superior.

En el caso de la columna $\mathrm{W}$, considerando que el valor del calificador es 0 , su sumatoria siempre será 0 . De igual forma su límite superior e inferior adoptará este valor. En el caso de la columna $\mathrm{X}$, que entrega 1 punto por celda, el mínimo valor es 1 (para una celda) y el máximo es 5 (para cinco celdas); por lo que $\Sigma$ puede fluctuar entre estos valores. En el caso de la columna Y, que entrega 2 puntos por celda, el mínimo valor es 2 (para una celda) y el máximo es 10 (para cinco celdas); por lo que $\Sigma$ puede fluctuar entre estos valores. Finalmente, en el caso de la columna $Z$, que entrega 3 puntos por celda, el mínimo valor es 3 (para una celda) y el máximo es 15 (para cinco celdas); por lo que $\Sigma$ puede fluctuar entre estos valores.

Se consideraron 4 categorías de atención, las que se detallan en la Tabla 2. Para determinar el rango de los puntajes se partió de la base que los niveles de complejidad están determinados por los calificadores y que estos van desde menor a mayor complejidad. De esta forma, y tal como se observa en la Tabla 2, el menor puntaje fue obtenido de la sustracción entre el límite superior y el límite inferior del respectivo categorizador; mientras que el mayor puntaje fue calculado de la sumatoria entre el límite superior del calificador correspondiente y un valor fijo asignado. Este valor fijo corresponde al valor de los categorizadores ordenados en forma decreciente. Es decir, para la primera categoría corresponde al máximo valor del calificador (3); para la segunda categoría corresponde al valor del calificador Y (2), y así sucesivamente.
Tabla 2. Obtención de los puntajes de corte para determinar las categorías de atención.

\begin{tabular}{|c|c|c|c|}
\hline Menor puntaje & Mayor Puntaje & Rango & Categoría \\
\hline (LsW - LiW) & $\mathrm{LsW}+3$ & $0-3$ & $\begin{array}{c}\text { No se justifica } \\
\text { atención }\end{array}$ \\
\hline$(\mathrm{Ls} X-\mathrm{LiX})$ & $\mathrm{LsX}+2$ & $4-7$ & Baja demanda \\
\hline (LsY - LiY) & $\mathrm{LsY}+1$ & $8-11$ & $\begin{array}{l}\text { Demanda } \\
\text { media }\end{array}$ \\
\hline (LsZ - LiZ) & $\mathrm{LsZ}+0$ & $12-15$ & Alta demanda \\
\hline
\end{tabular}

$\mathrm{LiW}=0 ; \mathrm{LsW}=0 ; \mathrm{LiX}=1 ; \mathrm{LsX}=5 ; \mathrm{LiY}=2 ; \mathrm{LsY}=10 ; \mathrm{LiZ}=3 ; \mathrm{LsW}=15$

\section{Función del Puntaje Total (PT)}

El valor del PT entrega la categoría, la carga terapéutica y la indicación terapéutica. En la Tabla 3 se expone dicha información.

Tabla 3. Carga terapéutica sugerida según categoría.

\begin{tabular}{|c|c|c|c|}
\hline Puntaje total & Categoría & Indicación & $\begin{array}{c}\text { Carga } \\
\text { terapéutica }\end{array}$ \\
\hline $0-3$ & $\begin{array}{c}\text { No se justifica } \\
\text { atención }\end{array}$ & - & - \\
\hline $4-7$ & Baja demanda & Control/Seguimiento & $\begin{array}{c}1 \text { o } 2 \text { sesiones } \\
\text { / semana }\end{array}$ \\
\hline $8-11$ & $\begin{array}{l}\text { Demanda } \\
\text { media }\end{array}$ & Rehabilitación & 1 sesión al día \\
\hline $12-15$ & Alta demanda & $\begin{array}{l}\text { Rehabilitación } \\
\text { intensiva }\end{array}$ & $\begin{array}{c}2 \text { sesiones al } \\
\text { día }\end{array}$ \\
\hline
\end{tabular}

\section{Carga terapéutica}

De acuerdo a la Tabla 3, cada categoría está asociada con una carga terapéutica, expresado en número de sesiones (diarias o semanales), para abordar al paciente con una frecuencia decreciente desde el mayor al menor nivel de complejidad. Es importante destacar que el manejo variará según la variable clínica que determina las mayores deficiencias del paciente. Por lo tanto, pueden existir pacientes de la misma complejidad, pero con diferentes requerimientos. Se consideran factores relevantes en la determinación de la frecuencia de las sesiones, desde el punto de vista clínico, el nivel de colaboración y/o la presencia de traqueostomía. 


\section{Aplicación y frecuencia}

La categorización se aplica luego de la evaluación inicial, donde se recomienda una valoración clínica integral que involucre cada área de atención fonoaudiológica.

El proceso de re-categorización dependerá de los avances del paciente en la terapia. Sin embargo, se aconseja aplicarla en forma semanal en centros con atención fonoaudiológica diurna y bisemanal en aquellos con disponibilidad de atención fonoaudiológica con modalidad de turnos o cobertura de fines de semana.

La aplicación de la matriz de categorización toma aproximadamente 1 minuto por paciente. El tiempo dependerá fundamentalmente del conocimiento que posee el clínico del paciente.

\section{Aspectos relevantes}

Es importante mencionar que la categorización busca orientar sobre la carga terapéutica requerida en base a las necesidades de los pacientes. Sin embargo, al ser una herramienta dinámica, podrán existir casos outliers que escapen del valor de la categorización, o que a criterio del clínico requieran mayor o menor carga terapéutica.

En ese sentido, y tal como fue mencionado en la sección "Carga terapéutica", existen factores relevantes desde la perspectiva clínica. Por tales motivos, nuestra herramienta se considera dos situaciones especiales:

- Aquellos sujetos con demanda media que sean usuarios de traqueostomía (TQT) y su nivel de cooperación sea moderada u óptima, automáticamente avanzan a alta demanda (rehabilitación intensiva). Esto se justifica en la necesidad de retiro rápido, efectivo y seguro de la vía aérea artificial, proceso en el que participan activamente los fonoaudiólogos. De acuerdo a datos del sistema GRD institucional, en el HUAP los pacientes traqueostomizados presentan un mayor peso GRD; lo cual implica además de mayor complejidad, mayor estadía hospitalaria, y por ende un mayor costo para la institución. Adicionalmente, el manejo de un paciente traqueostomizado en domicilio genera mayor carga para la familia y/o redes a cargo de sus cuidados. Estos antecedentes justifican el optimizar las atenciones de aquellos sujetos con TQT para contribuir en la decanulación. De esta forma, se busca disminuir la probabilidad de riesgo de infecciones, costos asociados a la atención en salud y carga futura del cuidador cuando el paciente reciba el alta.
- Aquellos sujetos con demanda media, pero con baja cooperación, caen en categoría baja demanda (control/seguimiento). Considerando el contexto del HUAP (alta demanda de rehabilitación fonoaudiológica y brecha de recursos humanos) es necesario optimizar las intervenciones en aquellos pacientes que posean un mayor potencial de rehabilitación activa, como es el caso de sujetos con buena cooperación. Por el contrario, por ejemplo, un paciente con diagnóstico de disfagia severa, que no cuenta con una adecuada conexión con el medio o carece de un nivel de cooperación moderada u óptima, no podrá participar activamente en su proceso de rehabilitación. En este caso se buscan estrategias preventivas o compensatorias, lo que implica una evolución más lenta. Sin embargo, es importante considerar que la rehabilitación es un proceso dinámico, por lo que al momento de re-categorizar podría existir una evolución favorable y mejorar la puntuación de la cooperación, lo que favorecerá la frecuencia de la carga terapéutica asignada.

\section{Confiabilidad}

La creación de la matriz de categorización incluyó un pilotaje que duró un mes. Este proceso tuvo como objetivo adaptarse al uso de la herramienta y pesquisar necesidades de ajustes en la misma, relacionado principalmente a estructura, variables u otros.

Durante el periodo de pilotaje y con el objetivo de establecer la fiabilidad inter-examinador de la matriz, se categorizaron 25 pacientes por dos examinadores en paralelo. Se calculó el coeficiente de correlación intraclase (CCI) utilizando un modelo combinado bidireccional y análisis de consistencia, obteniendo un valor de 0.9 , lo cual refleja una excelente fiabilidad interexaminador.

\section{Beneficios de su implementación}

El orden y la sistematización de los procesos de atención clínicoasistencial, constituyen una propuesta de gestión orientada a establecer mejoras en relación a procesos internos, uso eficiente de los recursos y estándares de calidad de la atención.

En nuestra experiencia, la implementación de esta matriz ha significado un beneficio en la gestión fonoaudiológica, pues ha permitido organizar y distribuir equitativamente la demanda asistencial, aun cuando no se contaba con los recursos humanos suficientes. También, ha posibilitado cuantificar el número total de atenciones fonoaudiológicas requeridas, así como su brecha, además de evidenciar el incremento en las atenciones. Los aspectos mencionados, sumado a otros instrumentos de gestión 
(por ejemplo, manuales de procedimientos o desarrollo de protocolos institucionales), han respaldado la justificación de incorporar nuevos profesionales, lo que impacta en el incremento de las atenciones y en la posibilidad de brindar mayor cobertura.

En este sentido, los datos internos de nuestro servicio dan cuenta de un incremento progresivo de la demanda de las atenciones, así como de los recursos humanos disponibles.

En el año 2018 nuestro servicio contaba con 2 fonoaudiólogos. Uno con jornada de 44 horas semanales (lunes a viernes) y otro por convenio asistencial-docente con jornada de 22 horas semanales, lo que se traduce en un total disponible de 66 horas semanales. En ese año se requerían en promedio 250 atenciones mensuales y se lograba un porcentaje de cumplimiento mensual de $50-65 \%$, lo que generaba una brecha de entre un $35-50 \%$ de las atenciones mensuales.

Contrastando esta información con el año 2020, existe un incremento significativo de los profesionales disponibles. Se cuenta con 5 fonoaudiólogos -todos dependientes de la institución-, los cuales se distribuyen en 4 fonoaudiólogos de 48 horas semanales (cobertura 12 horas diarias, los 7 días de la semana) y 1 de 44 horas semanales (lunes a viernes). Todo ello suma un total de 236 horas semanales disponibles. En términos porcentuales, al comparar esta información con la del año 2018, se evidencia un incremento aproximado de un $400 \%$ para la dotación profesional y de un $258 \%$ para la cantidad de horas semanales disponibles.

De igual forma, la demanda de atención fonoaudiológica se ha incrementado en forma significativa, requiriendo actualmente un promedio de 850 atenciones mensuales, lo que significa un incremento del $250 \%$ en comparación al año 2018. Además, se logra un porcentaje de cobertura mensual que va sobre el $95 \%$ y una brecha que es menor al $5 \%$.

\section{COMENTARIOS DE LOS AUTORES}

De acuerdo con lo propuesto por el Ministerio de Salud (MINSAL), las estrategias de rehabilitación precoz, intensiva y multidisciplinaria desde las Unidades de Paciente Crítico (UPC) buscan evitar complicaciones secundarias, acelerar los procesos de recuperación y mejorar la condición de alta; además de establecer estrategias que aseguren la derivación oportuna de personas que requieren continuar con su proceso de rehabilitación. Uno de los puntos cruciales para dar cumplimiento a estos objetivos es establecer la carga terapéutica y su perfil, lo cual dependerá de las condiciones generales de la persona, su patología de base, los objetivos acordados y el tiempo definido para alcanzarlos (MINSAL, 2018).

En este sentido, en el presente trabajo compartimos una herramienta clínica para valorar y estandarizar la carga de trabajo fonoaudiológico con pacientes adultos hospitalizados de un hospital público.

La información disponible respecto al número de atenciones necesarias y la frecuencia de estas, en la fase aguda o sub-aguda de hospitalización es escasa. Al respecto, lo más cercano en el contexto nacional es lo mencionado en el documento Modelo de Gestión Red de Rehabilitación (MINSAL, 2018), el cual es parte del plan de rehabilitación del MINSAL y plantea una frecuencia específica para atenciones fonoaudiológicas de entre 1 a 2 atenciones diarias, según el nivel de complejidad en rehabilitación del recinto hospitalario, enfatizando en la necesidad de ajustar la carga de tratamiento a las condiciones específicas de cada persona. Pese a ello, esta sugerencia parece más algo arbitraria y carece de un sustento teórico que avale la frecuencia de estas intervenciones, más si se considera la brecha de recursos humanos fonoaudiológicos que existe en los hospitales públicos.

La literatura avala el inicio precoz e intensivo de las intervenciones de rehabilitación con el objetivo de mejorar los outcomes funcionales (Arias-Fernández et al., 2018). Más allá de la frecuencia de las atenciones, que es un punto relevante, también debe ser considerada la dosis utilizada en las sesiones (intensidad, repeticiones, frecuencia, duración) y el tiempo desde el inicio del cuadro (Jette, 2017; Krekeler et al., 2020). A nivel fonoaudiológico, la evidencia sobre este aspecto es limitada y existe escasa información sobre la frecuencia y la dosis de las intervenciones (Baker, 2012). Este aspecto es crucial y amerita ser discutido, porque en varias ocasiones condiciona la frecuencia de las atenciones.

La rehabilitación fonoaudiológica precoz, intensiva, a altas dosis o durante un periodo prolongado produce mejoras funcionales en la comunicación de las personas con afasia (Brady et al., 2016). Del mismo modo, en etapas agudas, la rehabilitación fonoaudiológica intensiva, con frecuencia diaria, ha mostrado resultados beneficiosos en sujetos con afasia moderada-severa (Woldag et al., 2017). Pese a lo anterior, existe escaso consenso e insuficiente literatura sobre la frecuencia de las atenciones y de la dosis de intervención (Baker, 2012; Off et al., 2016). Ello hace que se desconozca el régimen óptimo de intervención para la rehabilitación de la afasia. Muchos estudios informan sobre la dosis de intervención, pero no entregan criterios sobre los contenidos activos dentro de las sesiones (Brady et al., 2016), lo 
cual puede generar diferencias sustanciales en relación con la eficiencia y eficacia de las intervenciones..

En aquellos casos de trastornos de habla adquiridos, la evidencia también es limitada respecto a la frecuencia de la intervención. Algunos estudios reportan mejorías significativas en los parámetros acústicos-perceptuales del habla, considerando sesiones de hasta 4 veces por semana (Mahler \& Ramig, 2012), mientras que otros consignan mejorías significativas en el grado de inteligibilidad del habla, considerando programas intensivos con hasta 2 sesiones diarias (Robertson, 2001). Si bien podría existir un efecto beneficioso inmediato, según el nivel de deterioro, la evidencia aún es limitada y se requieren mayores estudios sobre el tema (Mitchell et al., 2017).

Para los trastornos de la voz no se encontraron reportes referentes a frecuencia de las atenciones de terapia vocal en personas hospitalizadas. Sin embargo, esta condición es de interés considerando el alto riesgo de daño laríngeo secundario a periodos de ventilación mecánica invasiva, su vinculo estrecho con alteraciones de la deglución y su relevancia tanto en aspectos comunicativos como emocionales. En un estudio (Verdolini et al., 2012) que consideró sujetos con fonotrauma agudo y terapia de voz resonante, se evidenció que para reducir la inflamación de pliegues vocales la dosis de la terapia vocal debería ser a baja intensidad y corta duración (máximo 4 minutos). Si bien esta investigación no fue realizada en sujetos hospitalizados, sus resultados podrían extrapolarse. Los ejercicios debían ser repetidos varias veces al día, con al menos 15 minutos de descanso entre cada uno; siendo importante evaluar la habilidad del paciente en producir una voz sin esfuerzo antes de recomendar los ejercicios (Verdolini et al., 2012). La duración total de la terapia dependerá de la frecuencia de las sesiones, la severidad del cuadro, adherencia al tratamiento y capacidades del terapeuta. En general, se recomiendan 2 sesiones semanales, con una duración aproximada de 30 minutos. En la mayoría de los casos no se requieren más de 12 sesiones de terapia vocal para cumplir los objetivos (Murry \& Rosen, 2006). La valoración instrumental es fundamental para una adecuada intervención, siendo una limitación en nuestro contexto, debido a que en el HUAP no se cuenta con Otorrinolaringólogo.

Por su parte, en aquellos casos de alteraciones de la deglución, tanto el grado de severidad como el enfoque propuesto (compensatorio o reactivador), resultan determinantes al momento de plantear la frecuencia de las atenciones. Considerando la alta incidencia y prevalencia de la disfagia orofaríngea en el paciente hospitalizado y las consecuencias de este cuadro, que incrementan la morbi-mortalidad y los costos asociados a la atención en salud (Altman et al., 2010; Bakhtiyari et al., 2015; Moraes \& Andrade, 2011). La intervención fonoaudiológica precoz y de alta frecuencia es fundamental en este trastorno para lograr beneficios tempranos y prevenir complicaciones que pongan en riesgo la condición vital de la persona. Por lo tanto, la disfagia es una prioridad al momento de jerarquizar la intervención de las distintas alteraciones del ámbito fonoaudiológico. Al igual que en las otras áreas, se cuenta con escasa literatura que entregue información sobre métodos que condicionen la frecuencia de las intervenciones. Sin embargo, existe evidencia, aunque no es totalmente concluyente, relacionada con la dosis utilizada en diferentes ejercicios deglutorios (Clark, 2005; Krekeler et al., 2020). Esta evidencia podría servir como sustento al momento de establecer la frecuencia de las atenciones. Dependiendo del ejercicio y, según lo reportado, la frecuencia (entendida como número de series por día y números de días a la semana) podría fluctuar entre 3 veces/día por 3 días a la semana hasta 7 veces/día los 7 días de la semana. Las repeticiones (número de ejecuciones por serie) fluctúan entre 5 a 30 repeticiones por serie, con hasta 120 repeticiones por sesión. En relación con la intensidad (el porcentaje de máxima resistencia), escasos estudios reportaron esta medida en forma objetiva. Más bien se la definió como una ejecución sostenida, lo más fuerte posible o con máximo esfuerzo. Finalmente, las propuestas de duración total del programa de intervención variaron entre 4 semanas a 1 año (Krekeler et al., 2020).

Un aspecto importante para considerar en relación con lo propuesto en el documento del MINSAL (2018), es la diferencia entre la atención abierta y cerrada. En la propuesta ministerial, se plantea que en hospitales de mediana y alta complejidad, sobre un $70 \%$ de las atenciones sean realizadas en atención cerrada. Sin embargo, la intervención fonoaudiológica en el sistema de atención cerrada difiere sustancialmente de la atención abierta, desde el perfil de los pacientes hasta las prestaciones realizadas. Según lo reportado por la American Speech-Language-Hearing Association (ASHA), un $77 \%$ de las atenciones en pacientes adultos hospitalizados son de deglución, mientras que aproximadamente un $20 \%$ corresponden a atenciones por aspectos cognitivos y comunicativos (American Speech-LanguageHearing Association [ASHA], 2020). En este sentido, la atención prioritaria del trastorno deglutorio por sobre los trastornos de la comunicación se fundamenta en la importancia vital de la alimentación, en las complicaciones intrahospitalarias asociadas a su manejo deficitario y en los costos asociados a la atención en salud. Tales argumentos, como fue justificado en la selección de 
las variables clínicas, condicionaron su inclusión y relevancia en la matriz.

Es importante analizar la información previa de manera cuidadosa, pues la diferencia entre las atenciones de deglución versus las de comunicación podría estar condicionada por un subdiagnóstico en la pesquisa de trastornos de la comunicación, sumado a la brecha profesional existente, la priorización en el abordaje de la disfagia, el desconocimiento del resto de los profesionales en relación con el rol del fonoaudiólogo en el manejo de trastornos de comunicación. Lo anterior puede generar menores oportunidades de los fonoaudiólogos para satisfacer las necesidades de pacientes con déficits comunicativos debido a la presión del sistema de salud (Armstrong, 2003).

Adicionalmente, y como se mencionó, la presión hacia el sistema público de salud por mantener un balance positivo en la ocupación de camas, fomenta la toma de decisiones rápidas en el actuar clínico fonoaudiológico y condiciona muchas veces, la priorización de atenciones deglutorias por sobre las comunicativas.

Esta propuesta constituye un aporte para la práctica clínica de los fonoaudiólogos que se desempeñan con adultos en el contexto clínico-hospitalario. No hemos encontrado publicaciones a nivel nacional o internacional que entreguen orientaciones para desarrollar herramientas que determinen la carga terapéutica y la frecuencia de las atenciones. En este sentido, esta propuesta podría contribuir en mejorías de la gestión asistencial; así como en la estandarización de las atenciones fonoaudiológicas (referente a instrumentos de valoración clínica), además de ser una herramienta que permita justificar la brecha de atenciones y la necesidad de incrementar la dotación de recursos humanos fonoaudiológicos. Sin embargo, debe ser utilizada con precaución, puesto que la herramienta se aplica una vez el paciente es evaluado. Su uso es condicional a las derivaciones propias de cada centro y, por tanto, podrían existir casos subdiagnosticados.

En la experiencia en el HUAP, desde la incorporación oficial de Fonoaudiología el año 2017, la profesionalización de la disciplina ha permitido generar un modelo de trabajo autónomo, enfocado en la gestión y calidad de la atención, que ha demostrado la necesidad de incrementar atenciones fonoaudiológicas. En este sentido, la creación e implementación de la matriz de categorización se ha traducido en un beneficio, porque ha permitido fundamentar y justificar la brecha profesional, aumentando consecuentemente la dotación profesional, en base al incremento en las necesidades reales y objetivas de rehabilitación requeridas por nuestros pacientes. Otros beneficios evidenciados en la práctica clínica se relacionan con la disminución en los tiempos de acceso a Fonoaudiología (resolución de interconsultas), los cuales antiguamente podían tardar hasta 5 días hábiles. Actualmente, no tardan más de 48 horas. Por otra parte, los cambios implementados en la modalidad de trabajo (con posibilidad de coberturas los fines de semana y festivos) constituye una mejora en la continuidad del trabajo fonoaudiológico.

Se espera a futuro lograr establecer los indicadores de atención fonoaudiológica como metas de cumplimiento hospitalario, que contribuyan en los estándares de calidad de la atención y consecuentemente, sean considerados en los procesos de acreditación institucional.

A pesar de los beneficios del instrumento presentado, existen limitaciones importantes de analizar. En primer lugar, es fundamental destacar que el desarrollo de esta herramienta se basó sólo en la realidad local de una institución pública; por lo que al extrapolar su aplicación a otras instituciones de salud podrían existir diferencias vinculadas al contexto propio de cada hospital. Si bien las variables clínicas consideradas son transversales al ámbito hospitalario; podrían variar de acuerdo con las escalas de valoración utilizadas según los protocolos de cada institución. La limitación mencionada se intentó subsanar por medio de la descripción detallada del proceso de creación de la matriz de categorización y la asignación del puntaje. Con ello se busca que cualquier clínico pueda tomar esta información y adaptarla a su realidad local (por ejemplo, en casos que se utilicen otras escalas de valoración y/o se consideren otras variables clínicas de mayor relevancia para el contexto específico).

Una segunda limitación refiere a que esta herramienta carece de propiedades psicométricas que la validen. Si bien, no era el objetivo de esta investigación, calculamos su confiabilidad interexaminador en un proceso de pilotaje, la cual entregó valores de excelente concordancia. No obstante, no hemos realizado testeos referentes al cálculo de su validez de constructo y contenido.

Finalmente, se espera a futuro poder realizar investigaciones referentes a la validación de esta herramienta, con el objetivo de posicionarla como un instrumento aplicable en la práctica clínica fonoaudiológica nacional. Sin embargo, la propuesta actual, podría ser de ayuda para colegas que se desempeñen en el contexto hospitalario con el propósito de optimizar procesos de gestión, determinando brechas y justificando la contratación de nuevos profesionales fonoaudiólogos. 


\section{REFERENCIAS}

Altman, K. W., Yu, G.-P., \& Schaefer, S. D. (2010). Consequence of Dysphagia in the Hospitalized Patient: Impact on Prognosis and Hospital Resources. Archives of Otolaryngology-Head \& Neck Surgery, 136(8), 784-789. https://doi.org/10.1001/archoto.2010.129

American Speech-Language-Hearing Association [ASHA]. (2020). Getting Started in Acute Care Hospitals. American Speech-Language-Hearing Association; American Speech-Language-Hearing Association. https://www.asha.org/slp/healthcare/start_acute_care/

Arias-Fernández, P., Romero-Martin, M., Gómez-Salgado, J., \& FernándezGarcía, D. (2018). Rehabilitation and early mobilization in the critical patient: Systematic review. Journal of Physical Therapy Science, 30(9), 1193-1201. https://doi.org/10.1589/jpts.30.1193

Armstrong, E. (2003). Communication culture in acute speech pathology settings: Current issues. Advances in Speech Language Pathology, 5(2), 137-143. https://doi.org/10.1080/14417040510001669171

Baker, E. (2012). Optimal intervention intensity. International Journal of SpeechLanguage Pathology, 14(5), 401-409. https://doi.org/10.3109/17549507.2012.700323

Bakhtiyari, J., Sarraf, P., Nakhostin-Ansari, N., Tafakhori, A., Logemann, J., Faghihzadeh, S., \& Harirchian, M. H. (2015). Effects of early intervention of swallowing therapy on recovery from dysphagia following stroke. Iranian Journal of Neurology, 14(3), 119-124.

Brady, M. C., Kelly, H., Godwin, J., Enderby, P., \& Campbell, P. (2016). Speech and language therapy for aphasia following stroke. Cochrane Database of Systematic Reviews, 6. https://doi.org/10.1002/14651858.CD000425.pub4

Clark, M. (2005). Food for Thought. Perspectives on Swallowing and Swallowing Disorders (Dysphagia), 14(2), 24-27. https://doi.org/10.1044/sasd14.2.24

Daniels, S. K., Ballo, L. A., Mahoney, M. C., \& Foundas, A. L. (2000). Clinical predictors of dysphagia and aspiration risk: Outcome measures in acute stroke patients. Archives of Physical Medicine and Rehabilitation, 81(8), 1030-1033. https://doi.org/10.1053/apmr.2000.6301

De Jonghe, B., Sharshar, T., Lefaucheur, J.-P., Authier, F.-J., Durand-Zaleski, I., Boussarsar, M., Cerf, C., Renaud, E., Mesrati, F., Carlet, J., Raphaël, J.-C., Outin, H., Bastuji-Garin, S., \& Groupe de Réflexion et d'Etude des Neuromyopathies en Réanimation. (2002). Paresis acquired in the intensive care unit: A prospective multicenter study. JAMA, 288(22), 2859-2867. https://doi.org/10.1001/jama.288.22.2859

Duncan, E. A., \& Murray, J. (2012). The barriers and facilitators to routine outcome measurement by allied health professionals in practice: A systematic review. BMC Health Services Research, 12(1), 96. https://doi.org/10.1186/14726963-12-96

García, M. A., \& Castillo, L. (2000). Categorización de usuarios: Una herramienta para evaluar las cargas de trabajo de enfermería. Revista médica de Chile, 128(2), 177-183. https://doi.org/10.4067/S0034-98872000000200007

González-Seguel, F., Lee, J., Carcamo, M., Convalía, A., Merino, F., \& Ugarte, S. (2017). Functional Mobility in Mechanically Ventilated Critically ill Patients: An Observational Study. 1(2), 1-7.
Gouvier, W. D., Blanton, P. D., LaPorte, K. K., \& Nepomuceno, C. (1987). Reliability and validity of the Disability Rating Scale and the Levels of Cognitive Functioning Scale in monitoring recovery from severe head injury. Archives of Physical Medicine and Rehabilitation, 68(2), 94-97.

Gutiérrez-Panchana, T., \& Hidalgo-Cabalín, V. (2018). Adherence to standardized assessments through a complexity-based model for categorizing rehabilitation $\subset$ : Design and implementation in an acute hospital. BMC Medical Informatics and Decision Making, 18(1), 21. https://doi.org/10.1186/s12911-018-0590-1

Howard-Wilsher, S., Irvine, L., Fan, H., Shakespeare, T., Suhrcke, M., Horton, S., Poland, F., Hooper, L., \& Song, F. (2016). Systematic overview of economic evaluations of health-related rehabilitation. Disability and Health Journal, 9(1), 11-25. https://doi.org/10.1016/j.dhjo.2015.08.009

Jette, A. M. (2017). The Importance of Dose of a Rehabilitation Intervention. Physical Therapy, 97(11), 1043. https://doi.org/10.1093/ptj/pzx085

Krekeler, B. N., Rowe, L. M., \& Connor, N. P. (2020). Dose in Exercise-Based Dysphagia Therapies: A Scoping Review. Dysphagia. https://doi.org/10.1007/s00455-020-10104-3

Landeros, J., Quevedo, J., \& Rebolledo, V. (2014). Escala de Dependencia de Kinesiología: Un instrumento para la gestión. Kinesiología y Terapia Respiratoria. https://jmlanderos.wordpress.com/2014/04/16/escala-dedependencia-de-kinesiologia-un-instrumento-para-la-gestion/

Mahler, L. A., \& Ramig, L. O. (2012). Intensive treatment of dysarthria secondary to stroke. Clinical Linguistics \& Phonetics, 26(8), 681-694. https://doi.org/10.3109/02699206.2012.696173

Ministerio de Salud, División de Gestión de Redes Asistenciales [MINSAL]. (2018). Modelo de Gestión Red de Rehabilitación.

Mitchell, C., Bowen, A., Tyson, S., Butterfint, Z., \& Conroy, P. (2017). Interventions for dysarthria due to stroke and other adult-acquired, nonprogressive brain injury. Cochrane Database of Systematic Reviews, 1. https://doi.org/10.1002/14651858.CD002088.pub3

Moraes, D. P., \& Andrade, C. R. F. de. (2011). Indicadores de qualidade para o gerenciamento da disfagia em Unidades de Internação Hospitalar. Jornal da Sociedade Brasileira de Fonoaudiologia, 23(1), 89-94. https://doi.org/10.1590/S2179-64912011000100018

Murry, T., \& Rosen, C. (2006). The Role of the Speech-language Pathologist in The Treatment of Voice Disorders. En J. Rubin, R. T. Sataloff, \& G. Korovin (Eds.), Diagnosis and Treatment of Voice Disorders (pp. 575-584). Plural Publishing.

Off, C. A., Griffin, J. R., Spencer, K. A., \& Rogers, M. (2016). The impact of dose on naming accuracy with persons with aphasia. Aphasiology, 30(9), 983-1011. https://doi.org/10.1080/02687038.2015.1100705

O'Halloran, R., Worrall, L., \& Hickson, L. (2009). A psychometric investigation of speech, language and cognitive communicative rating scales for adults with acquired neurogenic communication disorders in the acute hospital setting. International Journal of Speech-Language Pathology, 11(3), 206-219. https://doi.org/10.1080/17549500902802298

O’Neil, K. H., Purdy, M., Falk, J., \& Gallo, L. (1999). The Dysphagia Outcome and Severity Scale. Dysphagia, 14(3), 139-145. https://doi.org/10.1007/PL00009595 
Radtke, J. V., Baumann, B. M., Garrett, K. L., \& Happ, M. B. (2011). Listening to the Voiceless Patient: Case Reports in Assisted Communication in the Intensive Care Unit. Journal of Palliative Medicine, 14(6), 791-795. https://doi.org/10.1089/jpm.2010.0313

Robertson, S. (2001). The efficacy of oro-facial and articulation exercises in dysarthria following stroke. International Journal of Language \& Communication Disorders, 36 Suppl, 292-297. https://doi.org/10.3109/13682820109177900

Steele, C. M., \& Cichero, J. A. Y. (2014). Physiological factors related to aspiration risk: A systematic review. Dysphagia, 29(3), 295-304. https://doi.org/10.1007/s00455-014-9516-y
Verdolini, K., Li, N. Y. K., Branski, R. C., Rosen, C. A., Grillo, E., Steinhauer, K., \& Hebda, P. A. (2012). Vocal exercise may attenuate acute vocal fold inflammation. Journal of voice : official journal of the Voice Foundation, 26(6), 814.e1-814.e13. https://doi.org/10.1016/j.jvoice.2012.03.008

Whyte, J., \& Kunz, R. (2018). Rancho Los Amigos Scale. En J. S. Kreutzer, J. DeLuca, \& B. Caplan (Eds.), Encyclopedia of Clinical Neuropsychology. Springer New York. https://doi.org/10.1007/978-0-387-79948-3

Woldag, H., Voigt, N., Bley, M., \& Hummelsheim, H. (2017). Constraint-Induced Aphasia Therapy in the Acute Stage: What Is the Key Factor for Efficacy? A Randomized Controlled Study. Neurorehabilitation and Neural Repair, 31(1), 72 80. https://doi.org/10.1177/1545968316662707 


\section{ANEXOS}

\section{Matriz de categorización de atenciones fonoaudiológicas}

\section{CATEGORIZACIÓN DE ATENCIÓN FONOAUDIOLÓGICA HOSPITALARIA}

Autor: Flgo. Axel Pavez Reyes

\begin{tabular}{|c|c|c|c|c|}
\hline VARIABLES & \multicolumn{4}{|c|}{ CALIFICADORES } \\
\hline $\begin{array}{l}\text { 1. Colaboración/ } \\
\text { Respuesta } \\
\text { (S5Q) }\end{array}$ & $\begin{array}{l}\text { Sin actividad / } \\
\text { No cooperador }\end{array}$ & $\begin{array}{c}\text { Baja } \\
\text { cooperación }\end{array}$ & $\begin{array}{c}\text { Cooperación } \\
\text { moderada }\end{array}$ & $\begin{array}{l}\text { Buena/óptima } \\
\text { cooperación }\end{array}$ \\
\hline Puntaje & 0 & 1 & 2 & 3 \\
\hline $\begin{array}{l}\text { 2. Funcionamiento } \\
\text { Cognitivo }{ }^{1} \\
\text { (ERLA) }\end{array}$ & $\begin{array}{l}\text { ERLA I-II / } \\
\text { No aplica }\end{array}$ & ERLA III-IV & ERLA V-VI & $E R L A \geq V I I$ \\
\hline Puntaje & 0 & 1 & 2 & 3 \\
\hline $\begin{array}{l}\text { 3. Trastorno de } \\
\text { deglución } \\
\text { (DOSS) }\end{array}$ & $\begin{array}{l}\text { Deglución funcional/ } \\
\text { Sin Trastorno }\end{array}$ & Disfunción leve & $\begin{array}{l}\text { Disfunción } \\
\text { moderada }\end{array}$ & Disfunción severa \\
\hline Puntaje & 0 & 1 & 2 & 3 \\
\hline $\begin{array}{l}\text { 4. Riesgo } \\
\text { aspirativo }\end{array}$ & Sin riesgo & Bajo riesgo & Riesgo moderado & Alto riesgo \\
\hline Puntaje & 0 & 1 & 2 & 3 \\
\hline 5. Comunicación & $\begin{array}{l}\text { Sin dificultad para } \\
\text { comunicarse }\end{array}$ & $\begin{array}{c}\text { Dificultad } \\
\text { exclusiva de } \\
\text { voz }\end{array}$ & $\begin{array}{c}\text { Dificultad de } \geq 1 \\
\text { componente (que } \\
\text { no sea voz) }\end{array}$ & $\begin{array}{l}\text { Disfunción de todo } \\
\text { ámbito comunicativo } \\
\text { (habla, voz, lenguaje) }\end{array}$ \\
\hline Puntaje & 0 & 1 & 2 & 3 \\
\hline
\end{tabular}

PTJE. OBTENIDO $\left({ }^{*}\right):$ _ 15
TQT: Si / No
DEMANDA:
No SESIONES:

CATEGORIZACIÓN SEGÚN CARGA DE

\begin{tabular}{l} 
TRABAO \\
\hline $12-15$ puntos: alta demanda $\rightarrow$
\end{tabular} rehabilitación intensiva ( 2 sesiones/día)

8-11 puntos: demanda media $\rightarrow$ rehabilitación $\left(1\right.$ sesión/día) ${ }^{(*)}\left({ }^{* *}\right)$

4-7 puntos: baja demanda $\rightarrow$ control/seguimiento (1-2 ses./semana)

0-3 puntos: no se justifica atención

(*) Pacientes con demanda media que sean usuarios de TQT y su nv. de cooperación sea moderada u óptima, automáticamente avanzan a alta demanda (rehabilitación intensiva)

$\left({ }^{* *}\right)$ Pacientes con demanda media pero con baja cooperación, caen en categoría baja demanda (control/seguimiento)

\begin{tabular}{|c|c|c|c|c|c|c|c|}
\hline \multirow{6}{*}{$\begin{array}{l}\quad \text { S5Q: } 5 \text { respuestas consecutivas } \\
\quad \text { (1 punto cada respuesta) } \\
\text { A. Abra y cierre sus ojos } \\
\text { B. Míreme } \\
\text { C. Abra su boca y saque la lengua } \\
\text { D. Asienta con la cabeza } \\
\text { E. Levante las cejas cuando cuente hasta } 5\end{array}$} & \multicolumn{4}{|c|}{$\begin{array}{l}\text { Escala del Funcionamiento Cognitivo } \\
\text { Rancho de los Amigos (ERLA) }\end{array}$} & \multicolumn{3}{|c|}{ Dysphagia Outcome and Severity Scale (DOSS) } \\
\hline & Nivel & Severidad & Asistencia & Puntaje & Nivel & Severidad & Puntaje \\
\hline & 1 & Sin respuesta & Total & 0 & 1 & Severa & 3 \\
\hline & II & Respuesta generalizada & Total & 0 & 2 & Moderada-Severa & 3 \\
\hline & III & Respuesta localizada & Máx. & 1 & 3 & Moderada & 2 \\
\hline & IV & Confuso / Agitado & Máx. & 1 & 4 & Leve-Moderada & 2 \\
\hline S5Q Correctas: $\quad / 5$ & \multirow[t]{2}{*}{$\mathrm{V}$} & \multirow{2}{*}{$\begin{array}{c}\text { Confuso / Inapropiado / } \\
\text { No agitado }\end{array}$} & \multirow[t]{2}{*}{ Máx. } & \multirow[t]{2}{*}{2} & 5 & Leve & 1 \\
\hline & & & & & \multirow[t]{4}{*}{6} & \multirow{4}{*}{$\begin{array}{l}\text { Dentro de límites } \\
\text { funcionales / } \\
\text { Independencia } \\
\text { modificada } \\
\end{array}$} & \multirow[t]{4}{*}{0} \\
\hline \multirow{5}{*}{$\begin{array}{l}\mathrm{S} 5 \mathrm{Q}=0 / 5 \rightarrow \text { sin actividad } / \text { no cooperador } \\
\mathrm{S} 5 \mathrm{Q}=1-2 \rightarrow \text { baja cooperación } \\
\mathrm{S} 5 \mathrm{Q}=3 \rightarrow \text { moderada cooperación } \\
\mathrm{S} 5 \mathrm{Q}=4-5 \rightarrow \text { buena/óptima cooperación }\end{array}$} & VI & Confuso / Apropiado & Mod. & 2 & & & \\
\hline & VII & Automático / Competente & Min. & 3 & & & \\
\hline & VIII & Determinado / Competente & Stand-By (S.B) & 3 & & & \\
\hline & IX & Determinado/Competente & S.B a solic. & 3 & \multirow[t]{2}{*}{7} & \multirow{2}{*}{$\begin{array}{l}\text { Dentro de límites de } \\
\text { normalidad }\end{array}$} & \multirow[t]{2}{*}{0} \\
\hline & $\mathrm{x}$ & Determinado / Competente & Indp. Modif. & 3 & & & \\
\hline
\end{tabular}

Riesgo Aspirativo

(basado en hallazgos fisiopatológicos y criterio clínico)

- Sin riesgo: pacientes que no presentan disfagia o mecanismo deglutorio funcional y su mecanismo tusígeno es efectivo.

- Bajo riesgo: pacientes con mecanismo funcional (nivel 6 DOSS) o disfagia, por general coincide con niveles 5 y 4 DOSS, que pueden presentar: episodios de voz húmeda o tos ocasional, con tos refleja efectiva, retención faringea que limpia espontáneamente (con subdegluciones o carraspeo), discreto retraso en activación de respuesta motora orofaríngea, discreta disminución en excursión hiolaríngea.

- Riesgo moderado: pacientes con disfagia, por lo general coincide con niveles 4 y 3 DOSS, que pueden presentar: sensibilidad intraoral disminuida con acumulación de residuos (pre y/o post deglución), deficitario control intraoral del bolo, episodios de penetración y aspiración consistentes ( $\geq 3$ para una misma consistencia), respuesta tusígena débil o parcialmente efectiva, sensibilidad faringolaríngea disminuida caracterizada por retención faríngea que debe ser limpiada con ayuda externa, retraso en activación de respuesta motora orofaríngea, disminución en excursión hiolaríngea, cierre glótico deficitario u otras según criterio del clínico.

- Alto riesgo: pacientes con disfagia, generalmente coincide con niveles 1 y 2 DOSS, que pueden presentar (además de los hallazgos mencionados en el punto anterior): tos débil, sensibilidad faringolaríngea disminuida, voz húmeda persistente que no logra limpiar en forma efectiva, sospecha de aspiración silente, incoordinación degluciónrespiración u otras según criterio del clínico.

${ }^{1}$ ERLA sólo aplicado en aquellos usuarios que presenten alguna limitación en su funcionalidad cognitiva (Se incluyen en este grupo pacientes ACV y trastornos cognitivos asociados, pacientes con TCC secundario a diversas etiologías -TEC, ACV, entre otros-, pacientes con alteración cognitiva, etc.)

Registro de propiedad intelectual $\mathrm{N}^{\circ}$ : 2020-A-7562 


\section{Ejemplos de uso}

A continuación, y para ejemplificar el modo de utilización de la matriz, se proponen algunos casos basados en antecedentes reales de pacientes que fueron categorizados.

\section{Caso 1:}

Hombre de 57 años de edad, sin antecedentes mórbidos relevantes. Ingresó con diagnóstico de TEC grave (GCS de ingreso 6 puntos). Requirió 11 días de intubación orotraqueal previa a la realización de TQT. Luego de 6 días posteriores a la realización de la TQT y consolidado el destete, se realizó evaluación fonoaudiológica inicial. Se aplicó matriz de categorización para determinar carga terapéutica, obteniendo los hallazgos descritos en la Tabla 4.

Tabla 4. Hallazgos clínicos y categorización de Caso 1.

\begin{tabular}{ccc}
\hline Variable & Calificador & Puntaje \\
\hline $\begin{array}{c}\text { Colaboración/Respuesta (S5Q) } \\
\text { Funcionamiento Cognitivo (ERLA) }\end{array}$ & Baja cooperación (S5Q 2/5) \\
Trastorno de Deglución (DOSS) & ERLA III & Disfunción severa (DOSS 1) \\
Riesgo aspirativo & Alto riesgo & 3 \\
Comunicación & Dificultad de $\geq 1$ componente (que no sea voz) & 2 \\
\hline
\end{tabular}

Interpretación: De acuerdo con los 10 puntos obtenidos, le correspondería ingresar a la categoría de demanda media, lo que implica indicación de rehabilitación con carga terapéutica de 1 sesión al día. Sin embargo, considerando el bajo nivel de cooperación y funcionamiento cognitivo en ERLA III, retrocede a categoría de baja demanda con indicación de control/seguimiento. Esto implica una carga terapéutica de 1-2 atenciones semanales hasta la re-categorización y mejoría clínica para la optimización de estas variables.

\section{Caso 2:}

Mujer de 71 años de edad, con antecedentes de Hipertensión y Diabetes. Ingresó con diagnóstico de Insuficiencia Respiratoria Aguda, requiriendo ventilación mecánica invasiva (VMI) por tubo orotraqueal durante 14 días. Una vez extubada y consolidado el weaning, se realizó evaluación fonoaudiológica inicial. Se aplicó matriz de categorización para determinar carga terapéutica, obteniendo los hallazgos descritos en la Tabla 5.

Tabla 5. Hallazgos clínicos y categorización de Caso 2.

\begin{tabular}{|c|c|c|}
\hline Variable & Calificador & Puntaje \\
\hline Colaboración/Respuesta (S5Q) & Moderada cooperación (S5Q 3/5) & 2 \\
\hline Funcionamiento Cognitivo (ERLA) & No aplica & 0 \\
\hline Trastorno de Deglución (DOSS) & Disfunción moderada (DOSS 3) & 2 \\
\hline Riesgo aspirativo & Moderado & 2 \\
\hline \multirow[t]{2}{*}{ Comunicación } & Dificultad exclusiva de voz & 1 \\
\hline & & 7 \\
\hline
\end{tabular}

Interpretación: De acuerdo con los 7 puntos obtenidos, ingresa a la categoría de baja demanda, con indicación de control/seguimiento, a la que le corresponde una carga terapéutica de 1-2 atenciones a la semana. 


\section{Caso 3:}

Hombre de 46 años, con antecedentes de policonsumo. Ingresó con diagnóstico de Politraumatismo (con trauma facial complejo). Requirió conexión a VMI por intubación orotraqueal durante 6 días. Ante el nivel de complejidad de su trauma facial y por necesidad quirúrgica se realizó TQT. A los 8 días de realizada la TQT logró weaning y se realizó evaluación fonoaudiológica inicial una semana después, luego de la autorización por el equipo de Cirugía Maxilofacial. Se aplicó matriz de categorización para determinar carga terapéutica, obteniendo los hallazgos descritos en la Tabla 6.

Tabla 6. Hallazgos clínicos y categorización de Caso 3.

\begin{tabular}{ccc}
\hline Variable & Calificador & Puntaje \\
\hline Colaboración/Respuesta (S5Q) & Buena/óptima cooperación (S5Q 4/5) \\
Funcionamiento Cognitivo (ERLA) & No aplica \\
Trastorno de Deglución (DOSS) & Disfunción severa (DOSS 1) \\
Riesgo aspirativo & Alto & 3 \\
Comunicación & Dificultad exclusiva de voz & 3 \\
\hline
\end{tabular}

Interpretación: De acuerdo con los 10 puntos obtenidos, le correspondería ingresar a la categoría de demanda media, lo que implica indicación de rehabilitación con carga terapéutica de 1 sesión al día. Sin embargo, considerando que se trata de una persona traqueostomizada y con buen nivel de cooperación, avanza a la siguiente categoría de alta demanda, lo que implica rehabilitación intensiva, con carga terapéutica de 2 atenciones al día. 